\title{
SHOULDER MUSCLE ELECTROMYOGRAPHIC ACTIVITY AND STIFFNESS IN PATIENTS WITH FROZEN SHOULDER SYNDROME: SIX-MONTH FOLLOW-UP STUDY
}

\author{
Jelena SokK ${ }^{1}$, Helena Gapeyeva ${ }^{1}$, JaAn Ereline ${ }^{1}$, Mati Merila ${ }^{2}$, \\ MATI PÄÄSUKE ${ }^{1}$ \\ ${ }^{1}$ Institute of Exercise Biology and Physiotherapy, University of Tartu, Tartu, Estonia \\ ${ }^{2}$ Department of Orthopaedics and Traumatology, Tartu University Hospital, Tartu, \\ Estonia
}

\begin{abstract}
This study evaluated changes in shoulder muscle isometric endurance, deltoideus and trapezius muscle electromyographic activity (EMG) and stiffness in patients with frozen shoulder syndrome (FSS) before and after manipulation under general anaesthesia (MUA). Eighteen FSS patients with mean age of $53 \pm 9$ years participated. Isometric endurance of shoulder muscles was characterized by endurance test time and deltoideus and trapezius muscles EMG activity that were assessed by electromyograph during weight holding in hand until exhaustion. Stiffness of deltoideus and trapezius muscles was assessed by myotonometer (MYOTON-3). Patients were screened by self-administered shoulder rating questionnaire (SRQ). Data was collected before one and six months after MUA. Six months after MUA endurance test time remained reduced $(\mathrm{p}<0.05)$ for the involved extremity as compared with the uninvolved extremity. Deltoideus and trapezius muscle EMG activity decreased $(\mathrm{p}<0.05)$ at the end of the endurance test, whereas in the beginning of the endurance test the trapezius muscle EMG was lower $(\mathrm{p}<0.05)$ for the involved extremity. Deltoideus and trapezius muscle stiffness did not differ $(\mathrm{p}<0.05)$. SRQ score points decreased $(\mathrm{p}<0.05)$ one and six months after MUA. In conclusion, six months after MUA the shoulder muscle EMG activity and stiffness for the involved extremity was normalized in patients with FSS.
\end{abstract}

Keywords: frozen shoulder syndrome, shoulder muscle endurance, shoulder muscle EMG activity, shoulder muscle stiffness 


\section{INTRODUCTION}

Frozen shoulder syndrome (FSS) occurs in $2-5 \%$ of the adult population [21] and up to $20 \%$ in those with diabetes [3], however, $60-65 \%$ of patients are women [33]. FSS is a long-lasting disease with an unclear etiology that goes through four stages, lasting from one to three years [28, 32], and requires patience from patients, doctors and physiotherapists. FSS patients have an increasingly severe shoulder pain and localized discomfort near the insertion of the deltoid muscle, limitation and pain in the active range of motion (AROM) and passive range of motion (PROM) especially in elevation and external rotation, and diminishing shoulder function in the sixth decade of life [27]. Shoulder girdle muscle strength has been found to be decreased in FSS patients $[14,15,18]$.

Electromyography (EMG) has been used to evaluate muscle function during or as a result of exercise $[13,22]$, muscle fatigue [2] and gait [6]. Some studies have investigated the shoulder muscle bioelectrical activity in patients with shoulder problems, whereas it was concluded that latissimus dorsi and pectoralis major are involved in the shoulder instability [13]. Lin et al. [19] found that patients with FSS compensate impaired glenohumeral motion by using accessory musculature. It was established that sustained trapezius muscle activity associated with neck and shoulder pain in healthy young subjects [12].

Leonard et al. [17] determined the correlations between myotonometric measurements of muscle stiffness and EMG at rest, during $6.8 \mathrm{~kg}$ weight holding isometrically and during maximal voluntary isometric contraction of the biceps brachii muscle. They found that myotonometer measurements of biceps brachii muscle stiffness highly correlated with EMG changes in healthy adults and that pathological change of muscle associated with various disabilities might demonstrate different correlation values. It has been found that myotonometer is a reliable tool for assessing muscle stiffness, tone and compliance of the relaxed or contracted biceps brachii and lateral gastrognemius muscles of nondisabled subjects [16]. Gavronski et al. [11] results support the use of MYOTON-2 as an objective method to evaluate the status of skeletal muscles, especially in sports medicine. Ditrolio et al. [8] found that myotonometry could detect changes in passive muscle stiffness in a number of clinical/therapeutic applications, whereas myotonometer is operated manually and requires minimal training.

To our best knowledge there are few studies that have assessed shoulder muscle electromyographic activity and stiffness in patients with FSS before and after MUA. We hypothesized that shoulder muscle electromyographic activity and stiffness differ for the involved and uninvolved extremity in patients with FSS before and after treatment. 


\section{MATERIALS AND METHODS}

\section{Subjects}

Eighteen patients with FSS ( 10 women and 8 men), aged 38 to 74 yrs participated in this study. The study was conducted at the University of Tartu, in the Laboratory of Kinesiology and Biomechanics. The subjects were recruited by orthopaedic surgeons in the Department of Orthopedics and Traumatology of Tartu University Hospital and their inclusion criteria were: unilateral FSS defined as: $>50 \%$ loss of pROM of the shoulder joint relative to the nonaffected side in 1 or more of 3 movement directions (i.e. ABD, FL, or EXR) $[7,35]$, shoulder pain at rest, inability to sleep on the affected side. Exclusion criteria were: previous MUA of the affected shoulder; other conditions involving the shoulder (rheumatoid arthritis, osteoarthritis, damage of the glenohumeral cartilage, Hill-Sachs lesion, osteoporosis or malignancies in the shoulder and chest region), traumatic bone or tendon changes in the affected shoulder; neurological deficits affecting shoulder function in ADL; shoulder pain or disorders of the cervical spine, elbow, wrist, or hand; and an injection with corticosteroids in the affected shoulder within 4 weeks. Patients who had had serious cardiac problems or cardiac surgery were excluded from the study [35].

The average duration from the onset of the disease to MUA was 8.6 months, ranging from 3 to 12 months. The FSS stage was II or III. The dominant shoulder was involved in 7 patients and the nondominant one in 11 patients. Three subjects did not return after the first session and were not included in data analysis. One subject broke the other hand one month after MUA. Two patients did not come back after the first session. The final data analysis was therefore conducted on 15 subjects. The subjects were moderately physically active, no professional athletes were included. They had no orthopaedic or neurological limitations or contraindications for exercise testing or training. All patients had physiotherapy about 7 times before MUA. Demographical data, including age, sex, employment status, and sports and leisure activities, were recorded at baseline. A history was taken concerning previous treatments (injections, physiotherapy), and current pain medications. Concomitant diseases, and the use of medications were registered. Subjects were informed about the procedures, and their written consent was obtained. The study carried the approval of the Ethics Committee of Human Studies of the University of Tartu. 


\section{Familiarization with tests}

The subjects were instructed and the shoulder muscle isometric endurance test was demonstrated 24-48 hours before collecting the first data. This was followed by a practical session to familiarize the subjects with the procedures. Before testing, each subject underwent a 10-min warm-up consisting of gymnastics, and stretching exercises. Both extremities were tested, whereas the uninvolved extremity was tested first. Data collection was performed preoperatively, and one and six months after MUA.

\section{Treatment}

The manipulation was done under general intravenous barbiturate anaesthesia for all patients. The following technique was used for manipulation: (a) gradual flexion (FL) in the sagittal plane to maximum possible extent while the surgeon's assistant fixed the scapula; (b) passive external rotation (EXR) was performed on 0 deg of abduction (ABD); (c) EXR in 90 deg of ABD; (d) internal rotation (INR) at $90 \mathrm{deg}$ of $\mathrm{ABD}$ and gross-body adduction (ADD) were performed. Care was taken not to fracture the humerus during manipulation. EXR force was very carefully applied when the patient's elbow was fixed and the wrist moved at the same time by the surgeon's thumb and two opposing fingers. A full shoulder ROM was always achieved. The shoulder joint was injected with $19 \mathrm{ml}$ of $1 \%$ Lidocaine and $1 \mathrm{ml}$ of corticosteroid in a $20 \mathrm{ml}$ syringe immediately after manipulation.

All patients received immediate passive exercise in the ward soon after MUA. They underwent gentle active-assisted motion with a physiotherapist after MUA. Motion was practiced during FL, extension (EXT), ABD, ADD, INR and EXR. Physiotherapy continued on an outpatient basis, and included supervised and therapeutic home exercise programmes focused on shoulder muscle stretching (two times per day, five days per week). Further physiotherapy procedures included shoulder muscle isometric strengthening exercises followed by the use of elastic bands and power simulator as soon as post-MUA shoulder pain and shoulder aROM allowed. The subjects were treated by physiotherapists with at least 2 years of clinical experience. The subjects had ten physiotherapy sessions during one month, three times per week. They were advised to use the affected shoulder in ADL whenever possible.

\section{Shoulder girdle muscle endurance test}

During shoulder muscles isometric endurance testing the subject was seated on standard chair, and the full extended upper extremity was positioned with the 
shoulder flexed $45^{\circ}$, and abducted $45^{\circ}$. In this position the subject hold weight in hand ( $5 \mathrm{~kg}$ for man and $3 \mathrm{~kg}$ for woman) until exhaustion.

\section{Electromyography}

During isometric endurance test electromyographic (EMG) activity of the deltoideus and trapezius muscles was continuously recorded using standard electromyograph Medicor MG 440 (Hungary). Paired bipolar surface electrodes (Ag-AgCl, 8-mm diameter, 20-mm interelectrode distance) were used. The skin under the electrode was shaved, abraded, and then cleaned with alcohol, and a conducting gel was applied to obtain a good signal transfer from the skin to the electrodes. The electrodes over the middle deltoid muscle halfway between the insertion and the acromion. The electrodes over the upper trapezius muscle were placed one third lateral and 2 to $4 \mathrm{~cm}$ above the line from processus $\mathrm{C} 7$ to acromion. The ground electrode was placed at the radial styloid process of the non-tested arm. Correct electrode placement was confirmed by observing the appropriate EMG activity while performing a manual muscle test.

The output signals from EMG preamplifiers were digitized online (sampling frequency $1 \mathrm{kHz}$ ) by analogue-to-digital converter installed in personal computer. The digitized signals were stored on a hard disk for further analyses, EMG power spectrum median frequency (MF) was calculated by using Fast Fourier Transform Algorithms, whereas a 1024 data point window ( $1 \mathrm{~s}$ ) slides over the whole recorded signal area with a 512 point shift ( $50 \%$ overlap). During shoulder muscle isometric endurance test the MF was determined, and averaged over each period of $5 \mathrm{~s}$, whereas the following characteristics were calculated: initial MF (MFi) as mean of the first $10 \mathrm{~s}$, and the mean of the last $10 \mathrm{~s}(\mathrm{MFe})$.

All measurements were rounded off to the nearest 5 degrees [35]. All assessments were performed by the same physiotherapist.

\section{Myotonometry}

Myotonometry and device MYOTON-3 elaborated at the University of Tartu was used for the assessment for visco-elastic properties (BP) of superficial skeletal muscles.

No investigations of viscous-elastic properties of shoulder muscles have been performed in patients with FSS. As a rule, the muscle of the left side of the body is measured first. During myotonometry assessment patient was supine on the investigation table. The patient should lie in a comfortable position and relax the muscles maximally during the measurement. 
The centre part of the deltoideus muscle and trapezius upper muscles in the middle part of muscle belly in MultiScan mode (10 times in each muscle with the interval of $1 \mathrm{~s}$ between measurements) were measured using myometer MYOTON-3 in lying position at rest [10].

\section{Shoulder rating questionnaire (SRQ)}

The functional limitation and disability of the shoulder was scored by the shoulder rating questionnaire (SRQ). The SRQ is a self-administered questionnaire including global assessment of shoulder pain, daily activities, areas of improvement and satisfaction, recreational and athletic activities, and work. The total score ranges from a minimum of 17 points (worst functional status) to a maximum of 100 points (best functional status) [20].

\section{Statistical analysis}

Data are presented as mean \pm SE and standard error \pm SE. One-way analysis of variance (ANOVA) followed by Bonferroni post hoc comparisons was used to evaluate differences between the involved and the uninvolved extremity. A paired $t$-test was used to evaluate differences between pre- and post-treatment characteristics. A level of $\mathrm{p}<0.05$ was selected to indicate statistical significance.

\section{RESULTS}

Before MUA a significant reduction $(\mathrm{p}<0.05)$ was noted for the involved extremity in the isometric endurance test time (Figure 1a) and in the EMG of deltoideus centre power spectrum MFi (Figure 1b) as compared with the uninvolved extremity. The deltoideus centre power spectrum MFi decreased significantly $(\mathrm{p}<0.05)$ during endurance test for both extremities, whereas trapezius upper power spectrum MFi (Figure 1c) decreasing was significant $(\mathrm{p}<0.05)$ for the uninvolved extremity. Muscle stiffness of deltoideus centre (Figure 2a) was significantly $(p<0.05)$ higher for the involved extremity as compared with the uninvolved extremity, whereas trapezius upper stiffness (Figure 2b) did not differ $(p>0.05)$ for both extremities. The endurance test time increased significantly $(\mathrm{p}<0.05)$ one and six months after MUA as compared with the pre-treatment level, whereas the involved extremity endurance time remained lower $(\mathrm{p}<0.05)$ as compared with the uninvolved extremity. 


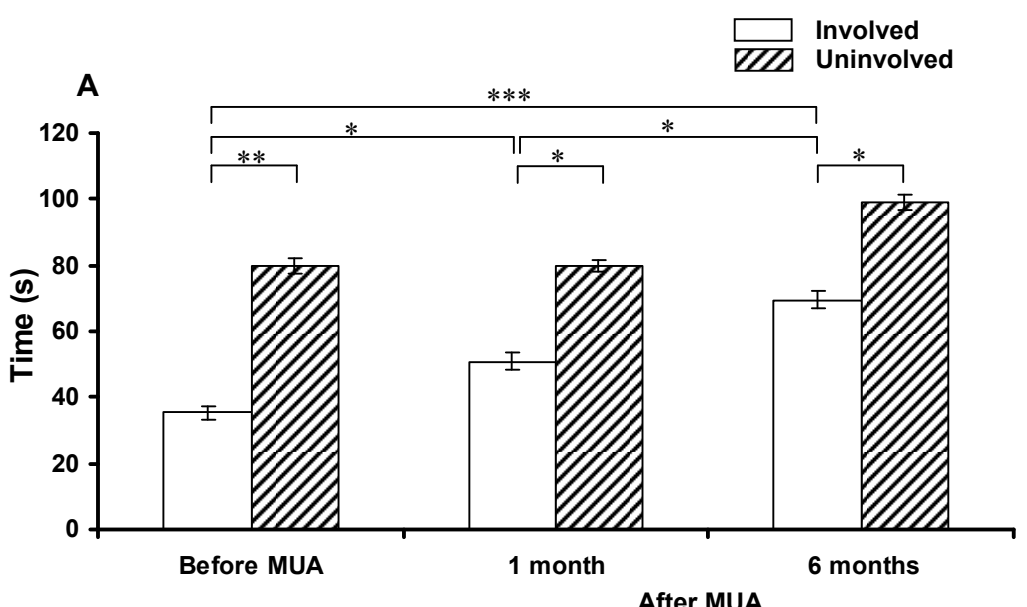

\section{B}

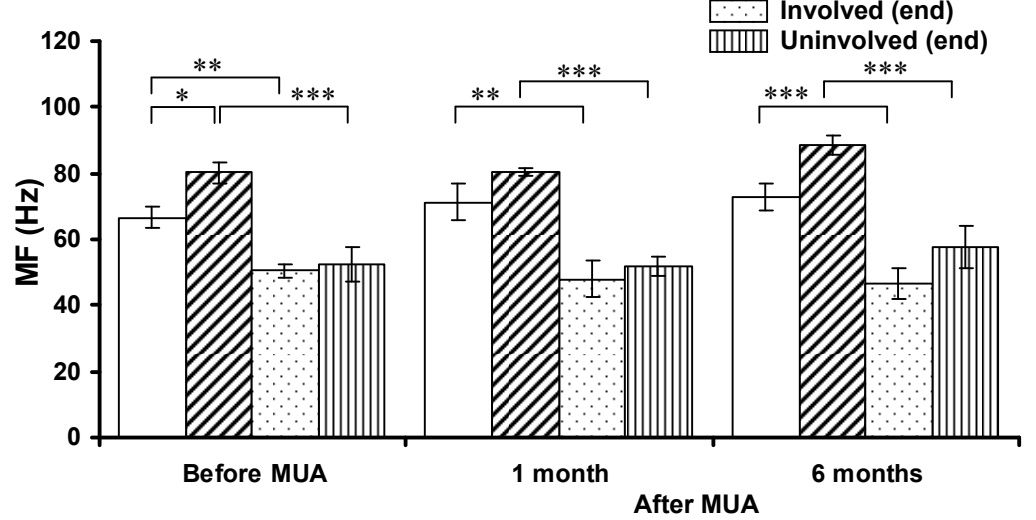

C

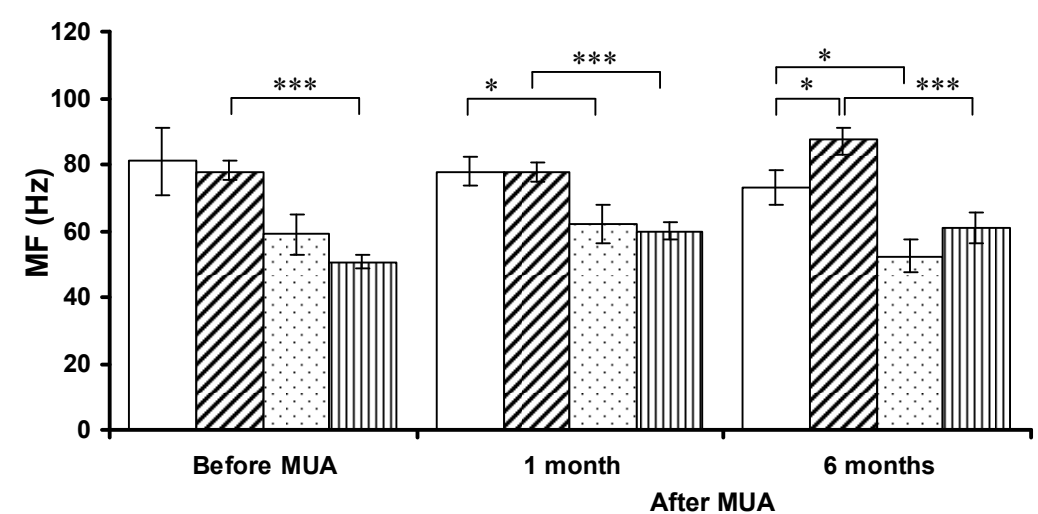

Figure 1. Mean $( \pm S E)$ shoulder muscle isometric endurance time (1a), power spectrum MF of deltoideus centre muscle (1b) and power spectrum MF trapezius upper muscle (1c) in patients $(n=15)$ with frozen shoulder syndrome (FSS) before and after manipulation under general anaesthesia (MUA) ${ }^{*} p<0.05,{ }^{* *} p<0.01$, ${ }^{* * *} p<0.001$. 


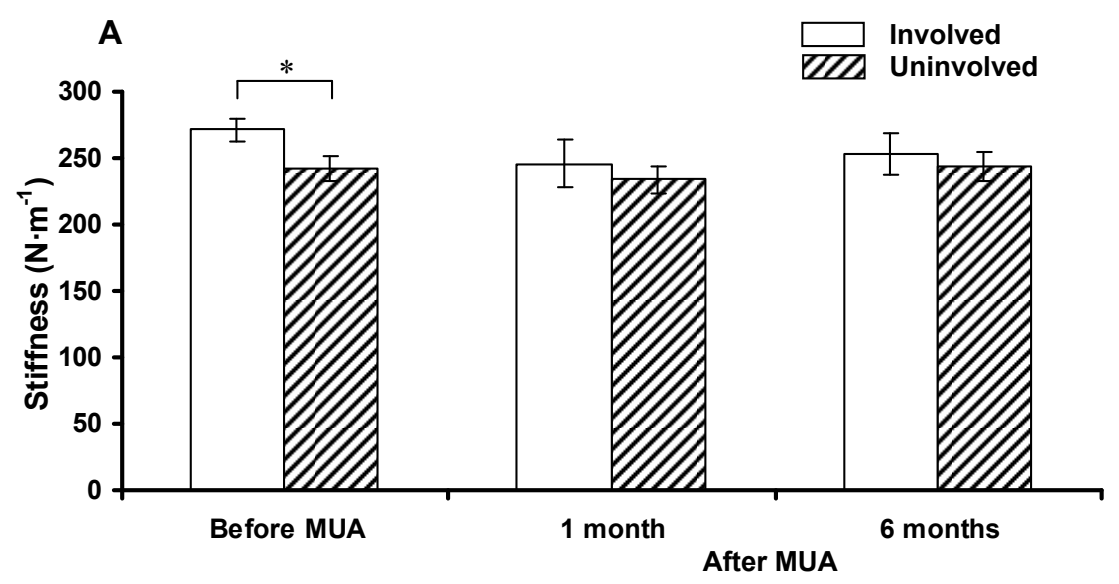

B

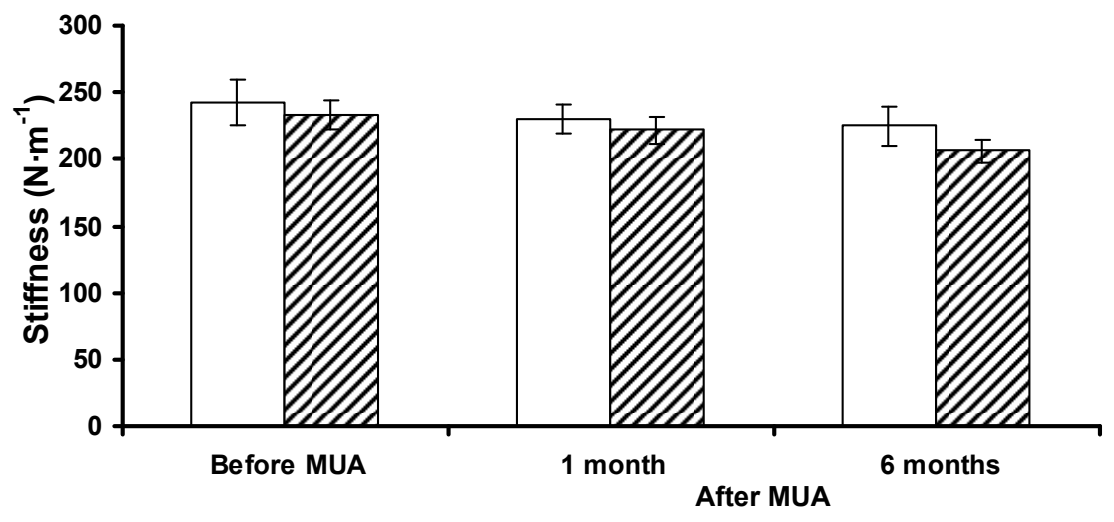

C

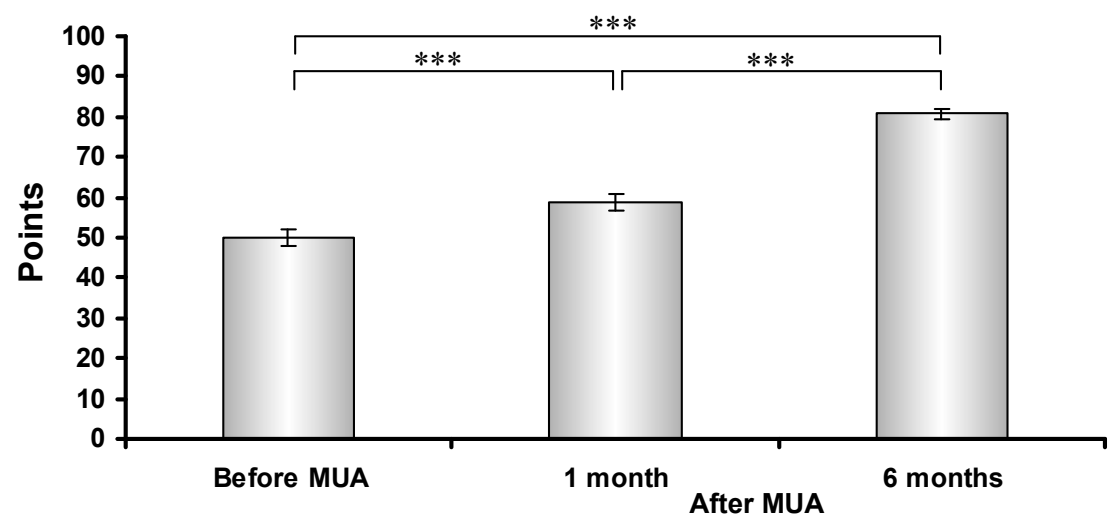

Figure 2. Mean $( \pm \mathrm{SE})$ muscle deltoideus centre stiffness (2a), muscle trapezius upper stiffness (2b) and shoulder rating questionnaire (2c) in patients $(n=15)$ with frozen shoulder syndrome (FSS) before and after manipulation under general anaesthesia (MUA) ${ }^{*} p<0.05,{ }^{* \star *} p<0.001$. 
The deltoideus centre and trapezius upper power spectrum MFi decreased significantly $(p<0.05)$ during endurance test for both extremities one and six months after MUA, whereas trapezius upper power spectrum MFi for the involved extremity was significantly $(\mathrm{p}<0.05)$ lower as compared with the uninvolved extremity six months after MUA. Six months after MUA muscle stiffness for the measured muscles did not differ significantly $(p>0.05)$. SRQ score points (Figure $2 c)$ increased significantly $(\mathrm{p}<0.05)$ one and six months after MUA.

\section{DISCUSSION}

The important finding of this study was that in patients with FSS the muscle EMG activity and stiffness of the deltoideus and trapezius upper muscles for the involved extremity approach the uninvolved extremity parameters one month after MUA.

The initial power spectrum MFi was $17 \%$ lower for the involved extremity of the deltoideus muscle as compared with the uninvolved extremity. The power spectrum MFi decreased at the end of endurance test $24 \%$ for the involved extremity and $34 \%$ for the uninvolved extremity. One month after MUA the power spectrum MFi for the deltoideus muscle increased 7\%. Roe et al. [30] found that during sustained submaximal contraction the EMG was increased for trapezius and deltoideus muscles in both affected and unaffected arm in patients with rotator tendionosis after supervised exercises which lasted over several months, whereas the fatigue development and recovery was unaltered by the exercises.

The power spectrum MF for the deltoideus and trapezius upper muscles decreased similarly for the both extremities one and six months after MUA. The trapezius muscle power spectrum MF decrease as compared to the initial level with the end point before MUA was $27 \%$ for the involved extremity and $35 \%$ for the uninvolved extremity, whereas six months after MUA the initial power spectrum MFi for the involved extremity was $16 \%$ lower.

After MUA the patients started with the physiotherapy programme as soon as possible and reported that the pain began to decrease one week after MUA. The pain decrease and shoulder function improvement led for the more active use of the involved extremity in everyday life. It is known that EMG activity decreases with painful stimulation during static and dynamic contractions [23]. Muscle pain instigates a decrease in the input to the motor neuron pool innervating the painful muscle, which causes reduced muscle activity [9]. Andresen et al. [1] concluded that in patients with trapezius myalgia emerged decreased strength capacity, and lowered activity of the painful trapezius muscle, whereas 
pain free deltoideus muscle activity was not significantly lower. Lin et al. [19] found that in patients with FSS the normalized EMG muscular activity for upper trapezius muscle was significantly higher during 60 deg abduction and flexion as compared with asymptomatic group. Adaptive posture may develop in patients with FSS, such as anterior shoulders or increased thoracic kyphosis [29]. So, in patients with FSS shoulder pain, long disease period and changed posture lead to the decreasing deltoideus and trapezius muscle EMG activity before MUA.

Stiffness of the deltoideus muscle was $11 \%$ higher for the involved extremity before and 5 and $4 \%$ for the involved extremity one and six months after MUA as compared with the uninvolved extremity. The deltoideus muscle stiffness decreased $10 \%$ one month and $7 \%$ six months after MUA for the involved extremity as compared with the pre-MUA level. Stiffness of the trapezius upper muscle was $4 \%$ higher for the involved extremity before MUA and one and six months after MUA 4 and $9 \%$ respectively. The trapezius upper muscle stiffness for the involved extremity decreased 5 and $7 \%$ one and six months after MUA as compared with the pre-MUA level. It was concluded that MYOTON-2 is a reliable tool for measuring the viscoelastic stiffness of resting muscle [4], myotonometry was also used in our study of patients with FSS. In the Viir et al. [36] study, the upper trapezius muscle stiffness in the supine position was $195 \mathrm{~N} / \mathrm{m}$ on the right side and $179 \mathrm{~N} /$ on the left side. In our study, these numbers were higher for the involved and uninvolved extremity before and six months after MUA and physiotherapy. This showed that pain increased muscle stiffness, whereas after MUA and physiotherapy sessions the involved extremity muscles stiffness parameters approach those of the uninvolved extremity. Marusiak et al. [24] concluded that myotonometer is a sensitive tool to assess passive stiffness of muscles in patients with Parkinson's disease as compared with healthy controls. Patients with Parkinson's disease have higher values of passive stiffness of the muscle belly and tendon [25].

Shoulder rating questionnaire point score increased significantly after MUA and physiotherapy as compared with the pre-MUA level. Vad et al. [34] investigated the role of capsular distension in patients with FSS and found that SRQ scores improved after treatment. Patients' endurance test time remained reduced six months after MUA, whereas the endurance test time increase was $31 \%$ and six months $49 \%$ as compared with the pre-MUA level. It was found that patients with temporomandibular disorders had a significantly reduced endurance test time for cervical extensor muscles as compared with healthy controls [2]. It was found that in patients with unilateral rotator tendinosis shoulder muscle fatigue development and recovery was unaltered by the 
3-6-month supervised exercise regimen [30] whereas Brox et al. [5] stated that endurance time during the submaximal contraction were not affected by pain. Pain and patients' motivation are important factors for the muscle endurance testing.

In conclusion, the results of this study show that in patients with FSS before MUA the deltoideus muscle EMG activity, muscle stiffness and muscle endurance time were lower, and functional limitation was higher for the involved extremity, whereas all measured parameters normalized for the involved extremity six months after MUA except endurance time. The muscle EMG activity and muscle stiffness measurement provide doctors and physiotherapists with additional information about the involved shoulder muscle condition.

\section{ACKNOWLEDGEMENTS}

This study was partly supported by Estonian Ministry of Education and Research project No SF0180030s07 and Estonian Science Foundation project No 7939.

\section{REFERENCES}

1. Andresen LL, Nielsen PK, Sogaard K, Andresen CH, Skotte J, Sjogaard G. (2008) Torque-EMG-velocity relationship in female workers with chronic neck muscle pain. J Biomech, 41: 2029-2035

2. Armijo-Olivo S, Silvestre RA, Fuentes JP, de Costa BR, Major PW, Warren S, Thive NM, Magee DJ. (2012) Patients with temporomandibular disorders have increased fatigability of the cervical extensor muscles. Clin J Pain, 28: 55-64

3. Balci N, Balci MF, Tüzüner S. (1999) Shoulder adhesive capsulitis and shoulder range of motion in type II diabetes mellitus: associations with diabetic complications. J Diabetes Complications, 13: 135-140

4. Bizzini M, Mannion AF. (2003) Reliability of a new, hand-held device for assessing skeletal muscle stiffness. Clin Biomech, 18: 459-461

5. Brox JI, Roe C, Saugen E, Vollestad NK. (1997) Isometric abduction muscle activation in patients with rotator tendionosis. Arch Phys Med Rehabil, 78: 1260-1267

6. Cenni F, Leardini A, Pieri M, Berti L, Belvedre C, Romagnoli M, Gianni S. (2013) Functional performance of a total ankle replacement: thorough assessment by combining gait and fluoroscopic analyses, 28: 79-87

7. Diercks RL, Stevens M. (2004) Gentle thawing of the frozen shoulder: a prospective study of supervised neglect versus intensive physical therapy in seventy-seven 
patients with frozen shoulder syndrome followed up for two years. J Shoulder Elbow Surg, 13: 499-502

8. Ditroilo M, Hunter AM, Haslam S, De Vito G. (2011) The effect of two novel techniques in establishing the mechanical and contractile responses of biceps femoris. Physiol Meas, 32: 1315-1326

9. Falla D, Arendt-Nielsen L, Farina D. (2009) The pain-induced change in relative activation of upper trapezius muscle regions is independent of the site of noxious stimulation. Clin Neurophysiol, 12: 150-157

10. Gapeyeva H, Vain A. (2008) Principles of Applying Myoton in Physical Medicine and Rehabilitation. Methodical guide. Müomeetria Ltd.

11. Gavronski G, Versakstis A, Vasar E, Maaroos J. (2007) Evaluation of viscoelastic parameters of the skeletal muscles in junior triathletes. Physiol Meas, 28: 625-637

12. Hanvold TN, Wærsted M, Mengshoel AM, Bjertness E, Stigum H, Twisk J, Veiersted KB. (2013) The effect of work-related sustained trapezius muscle activity on the development of neck and shoulder pain among adults. Scand J Work Environ Health, doi:10.5271/sjweh.3357

13. Jaggi A, Noorani, Malone A, Cowan J, Lambert S, Bayley I. (2012) Muscle activation patterns in patients with recurrent shoulder instability. Int J Shoulder Surg, 6: 101-107

14. Jürgel J, Rannama L, Gapeyeva H, Ereline J, Kolts I, Pääsuke M. (2005) Shoulder function in patients with frozen shoulder before and after 4-week rehabilitation. Medicina (Kaunas), 41:30-8

15. Kanbe K, Inoue K, Inoue Y, Chen Q. (2009) Inducement of mitogen-activated protein kinases in frozen shoulders. J Orthop Sci, 14: 56-61

16. Leonard CT, Deshner WP, Romo JW, Suoja ES, Fehrer SC, Mikhailenok EL. (2003) Myotonometer intra- and interrater reliabilities. Arch Phys Med Rehabil, 84: 928-932

17. Leonard CT, Brown JS, Price TR, Queen SA, Mikhailenok EL. (2004) Comparison of surface electromyography and myotonometric measurements during voluntary isometric contractions. J Electromyogr Kinesiol, 14: 709-714

18. Lin HS, Li JS, Lo SF, Shin YF, Lo CY, Chen SY. (2009) Isokinetic characteristics of shoulder rotators in patients with adhesive capsulitis. J Rehabil. Med, 41: 563-568

19. Lin JJ, Wu YT, Wang SF, Chen SY. (2005) Trapezius muscle imbalance in individuals suffering from frozen shoulder syndrome. Clin Rheumatol, 24: 569-575

20. L'Insalata JC, Warren RF, Cohen SB. (1997) A self-administered questionnaire for assessment of symptoms and function of the shoulder. J Bone Joint Surg Am 79: $738-748$

21. Lorbach O, Anagnostalos K, Scherf C, Seil R, Kohn D, Pape D. (2010) Nonoperative management of adhesive capsulitis of the shoulder: oral cortisone applications versus intra-articular cortisone injections. J Shoulder Elbow Surg, 19: 172-179

22. Ludewig PM, Hoff MS, Osowski EE, Meschke SA, Rundquist PJ. (2004) Relative balance of serratus anterior and upper trapezius muscle activity during push-up exercises. Am J Sports Med, 32: 484-493 
23. Lund JP, Donga R, Widmer CG, Stohler CS. (1991) The pain-adaption model: a discussion of the relationship between chronic musculoskeletal pain and motor activity. Can J Physiol Pharmacol, 69: 683-694

24. Marusiak J, Kisiel-Sajewicz K, Jaskolska A, Jaskolski A. (2010) Higher muscle passive stiffness in Parkinson's disease patients than in controls measured by myotonometry. Arch Phys Med Rehabil, 91: 800-802

25. Marusiak J, Jaskolska A, Budrewicz S, Koszewicz M, Jaskolski A. (2011) Increased muscle belli and tendon stiffness in patinest with Parkinsons disease, as measured by myotonometry. Mov Dis, 26: 2119-2122

26. McDonald A, Picco BR, Belbeck AL, Chow AY, Dickerson CR. (2012) Spatial dependency of shoulder muscle demands in horizontal pushing and pulling. Appl Ergon, 43: 971-978

27. Neviaser RJ, Neviaser TJ. (1987) The frozen shoulder. Diagnosis and management. Clin Orthop Relat Res, 223: 59-64

28. Neviaser AS, Hannafin JA. (2010) Adhesive capsulitis: a review of current treatment. Am J Sports Med, 38: 2345-2356

29. Page P, Labbe A. (2010) Adhesive capsulitis: use the evidence to integrate your intervention. N Am J Sports Phys Ther, 5: 266-273

30. Roe C, Brox JI, Bohmer AS, Vollestad NK. (2000) Muscle activation after supervised exercises in patients with rotator tendionosis. Arch Phys Med Rehabil, 81: 67-72

31. Rose SA, Õunpuu S, DeLuca PA. (1991) Strategies for the assessment of pediatric gait in the clinical setting. Phys Ther, 71: 961-980

32. Shaffer B, Tibone JE, Kerlan RK. (1992) Frozen shoulder. A long-term follow-up. J Bone Joint Surg Am, 74: 738-746

33. Sheridan MA, Hannafin JA. (2006) Upper extremity: emphasis on frozen shoulder. Orthop Clin N Am, 37: 531-539

34. Vad VB, Sakalkale D, Warren RF. (2003) The role of capsular distension in adhesive capsulitis. Arch Phys Med Rehabil, 84: 1290-1292

35. Vermeulen HM, Obermann WR, Burger BJ, Kok GJ, Rozing PM, van Den Ende $\mathrm{CH}$. (2000) End-range mobilization techniques in adhesive capsulitis of the shoulder joint: A multiple-subject case report. Phys Ther, 80: 1204-13

36. Viir R, Virkus A, Laiho K, Rajaleid K, Selart A, Mikkelsson M. (2007) Trapezius muscle tone and viscoelastic properties in sitting and supine positions. SJWEH Suppl, 3: 76-80

\section{Correspondence to:}

Jelena Sokk

Institute of Exercise Biology and Physiotherapy,

University of Tartu

Jakobi 5, 51014, Tartu, Estonia

Tel./fax: +37-27-376286

E-mail: jelena.sokk@ut.ee 